\title{
High-efficiency non-viral transfection of human primary $T$ lymphocytes
}

Ilnaz Rahimmanesh ${ }^{1}$, Mehdi Totonchi ${ }^{2}$, Hossein Khanahmad $^{1 *}$

1- Department of genetics and molecular biology, School of Medicine, Isfahan University of Medical Sciences, Isfahan, Iran

2- Department of Stem Cells and Developmental Biology, Royan Institute for Stem Cell Biology and Technology, ACECR, P.O. Box 19395-4644, Tehran, Iran.

\section{*Corresponding author: Dr. Hossein Khanahmad}

Isfahan University of Medical Sciences, School of Medicine, Department of Genetics and Molecular Biology, Isfahan, Iran.

Phone: +98-313-7929144

Fax number: +98-313-6688597

H khanahmad@med.mui.ac.ir

\section{Abstract:}

The development and optimization of an effective non-viral gene delivery method for genetic manipulation of primary human $\mathrm{T}$ cells is a major challenge in clinical immunotherapy researches. According to the low transfection efficiency of conventional methods in human primary $\mathrm{T}$ cells, there is an effort in order to increase the transfection rate in these cells. Protamine is an FDA-approved compound with a documented safety profile that enhances DNA condensation for gene delivery. In 
this study, the effect of protamine on the transfection efficiency of standard transfection reagents, TurboFect, and Lipofectamine 2000 was evaluated in order to transfect primary human $\mathrm{T}$ cells. Results demonstrated that protamine condenses DNA and increases the positive charge of DNA/Cargo complex efficiently without any cytotoxic effect on the primary human $\mathrm{T}$ cells. The results also revealed that the DNA/Protamine/Cargo complexes effectively transfect human primary T cells. Keyword: Human primary T cells, Gene delivery, Protamine sulfate, Transfection

\section{Introduction:}

The vast clinical success of chimeric antigen receptor (CAR) T cell immunotherapy in targeting various blood malignancies and solid tumors, and the application of other $\mathrm{T}$ cell subsets in the treatment of autoimmune diseases has created an inevitable need for a cost effective, rapid and efficient method for ex-vivo gene delivery to primary human T cells (1). Recently, most of clinical trials applied gamma-retroviral and lentiviral methods for genetic manipulation of $\mathrm{T}$ cells led to permanent CAR expression (2-4).

Although viral vectors are very efficient, they are costly to produce at the GMP scale and have significant limitations such as cytotoxicity, immunogenicity and insertional mutagenesis for clinical applications (5). 
Non-viral methods including electroporation and chemical transfection reagents have been suggested as alternative strategies for gene delivery to mammalian cells. However, several studies have proven the challenging nature of primary $\mathrm{T}$ lymphocytes manipulation using non-viral gene delivery systems due to low transfection efficiency rate and the transient expression of introduced gene, as the most important limitations $(6,7)$.

Since $\mathrm{T}$ lymphocytes are considered as a hard to transfect primary cell, electroporation is becoming the most commonly non-viral method used for gene delivery to $\mathrm{T}$ lymphocytes. Electroporation is an efficient physical method to introduce a gene into the cells; however, electroporation conditions need to be optimized because of low transfection efficiency and poor cellular viability, specifically in primary human lymphocytes $(8,9)$.

On the other hand, T cells are resistant to common chemical transfection reagents which have a safety profile for many mammalian cells, such as Lipofectamine 2000, polyfect and TurboFect. These reagents are less popular in the case of $\mathrm{T}$ cell transfection due to their low transfection efficiency (10).

Therefore, improvement of non-viral gene delivery methods is required for an effective gene transfer into T cells, as well as reduction of carrier's cell toxicity.

There are numerous obstacles to develop a promising non-viral gene delivery strategy including DNA/carrier complex formation, cell entrance mechanism, 
endosomal escape, dissociation of the DNA from the cargo and nuclear translocation $(6,7)$

In order to promote the transfection process, it is desired to facilitate the electrostatic interaction of the transfection reagent with the negative charge of DNA backbone and the cell membrane (11). Also, previous data has been shown that precondensation of DNA by an additional cationic charge improves its protection against degradation (12).

Protamines are small peptides (MW 4-10 kDa), FDA approved cationic nuclear protein enriched in arginine. Due to their membrane-translocating activity and the ability to improve DNA packaging, they are widely studied in the gene delivery methods. Protamines are isolated from the sperm of mature fish (13). In fertilization procedure, protamines bind to and condense genomic DNA, and deliver DNA to the nucleus due to containing four nuclear localization sequences (NLS) in their structure (14).

This unique role of protamine which exactly straight forwards the DNA complex to the nucleus is desirable for overcoming some major hurdles in gene delivery.

In this study, the effect of protamine on the transfection efficiency of standard transfection reagents, TurboFect and Lipofectamine 2000, was evaluated in order to transfect primary human T cells. 


\section{Methods}

\section{Isolation of PBMC and T-cell culture:}

Peripheral blood mononuclear cells (PBMCs) were isolated from heparinized blood using the Ficoll-hypaque density gradient. The isolated cells were incubated for 24 hours in complete RPMI-1640 (Bioidea, Iran) medium supplemented with 10\% FBS (Bioidea, Iran) at $37^{\circ} \mathrm{C}$ in a humidified incubator supplied with $5 \% \mathrm{Co}_{2}$ to isolate monocytes in $75 \mathrm{~T}$ cell culture flask. Lymphocytes were collected at $500 \times \mathrm{g}$ for 5 minutes and suspended in complete RPMI-1640 medium containing $1 \mu \mathrm{g} / \mathrm{ml}$ phytohemagglutinin (Sigma, USA) and then cells were incubated for $24 \mathrm{~h}$ at $37^{\circ} \mathrm{C}$ and $5 \% \mathrm{Co}_{2}$. T cells were expanded using $3.3 \mu \mathrm{g} / \mathrm{ml}$ anti-CD3 monoclonal antibody (Biolegend, USA) according to manufacturer's instruction.

\section{Cell lines:}

The Jurkat cells (human leukemia T cells) and Human Embryonic Kidney 293 cells (HEK-293) were purchased from Pasteur institute (Tehran, Iran) and maintained in complete RPMI-1640 medium and cultured at $37{ }^{\circ} \mathrm{C}$ in a $5 \% \mathrm{CO}_{2}$ humidified incubator.

\section{Preparation of Plasmid/Protamine Complexes and detection of electrostatic binding of protamine to DNA:}

The plasmid DNA, pCDH513-B, encoding green fluorescent protein (GFP) was extracted from E.coli $\mathrm{TOP} 10 \mathrm{~F}^{\prime}$ and purified using plasmid extraction kit according 
to manufacturer's protocol (Genetbio, Korea). The purity of the extracted plasmid was checked by electrophoresis on a $1 \%$ agarose gel and the concentration of DNA was estimated by measuring the absorbance at $260 \mathrm{~nm}$.

One microgram of purified plasmid was mixed with different amounts of protamine sulfate (Sigma, USA) to achieve the following N/P ratios: 20:1, 10:1, 5:1, 3:1, 2:1 and 1:1 in $150 \mathrm{mM} \mathrm{NaCl}$. The mixtures were incubated at room temperature for 15 $\min$.

After incubation, the binding of protamine to DNA in each mixture was evaluated using a dye displacement assay and the electrophoretic mobility (15 min at $120 \mathrm{~V}$ ) of the mixture was visualized on $1 \%(w / v)$ agarose gel stained with EcoDye (BIOFACT, Korea) to detect the optimal DNA/Protamine ratio.

\section{The zeta potential of DNA/Protamine/Cargo Complexes:}

To detect the surface charge of different DNA/Cargo complexes, which was prepared by adding of different transfection regents to optimal DNA/Protamine ratio, the value of the zeta potential was determined using Laser Zeta meter (HORBIO Scientific-Japan) in water solution $(\mathrm{pH}=7.4)$.

\section{T cells transfection:}

Primary T cells and Jurkat cells, as well as HEK-293 as the control, were seeded in 24-well plates at a density of $2 \times 10^{5}$ approximately $24 \mathrm{~h}$ before transfection procedure which was performed in triplicate. Then $2.5 \mu \mathrm{L}$ of transfection reagents, 
TurboFect and Lipofectamine 2000 (Thermo Fisher Scientific, USA), were added to the optimal DNA/Protamine complex, prepared as described earlier, and incubated for additional $15 \mathrm{~min}$ at room temperature.

The mixtures were subsequently added to the cells and transfection procedure carried out according to manufacturer's instruction. The cells which were transfected using transfection reagents alone, served as controls.

\section{Transfection efficiency measurement:}

Cells were then harvested $24 \mathrm{~h}$ after transfection in order to evaluate the transfection efficiency in each group using fluorescent microscopy and flowcytometry analysis in parallel. Cells were at $500 \times \mathrm{g}$ for $5 \mathrm{~min}$ and then washed with phosphate buffer saline (PBS) (Bioidea, Iran). The GFP expression was evaluated with BD flow cytometr (BD Bioscience, USA) by accumulating up to 100,000 cells per tube and the obtained data were analyzed by Cell Quest software version 8.6.1 (BD Bioscience, USA).

\section{Real-time polymerase chain reaction}

Total RNA of about $2 \times 10^{5}$ transfected T cells was extracted using RNX kit (Cinagen, Iran) and treated with DNase I enzyme (Thermo Scientific, USA). The quality and quantity of the extracted RNA was assessed spectrophotometrically and by electrophoresis. First strand cDNA was synthesized using $2 \mu \mathrm{g}$ of the total RNA according to first strand cDNA synthesis kit instruction (Thermo Scientific, USA). 
Relative quantification was performed using following specific primers: GFP forward, 5'-AAGCTGACCCTGAAGTTCATCTGC-3', and GFP reverse, 5'CTTGTAGTTGCCGTCGTCCTTGAA-3', Actin forward, 5'ATGTGTGACGAAGAAGCATCAGCC-3', and Actin reverse, 5'TCATCCCAGTTGGTGATAATGCCG -3'.

All real-time RT-PCR reactions were prepared in triplicate by mixing $10 \mu \mathrm{L}$ of SYBR green master mix (Ampliqon kit, Denmark), $0.5 \mu \mathrm{L}$ of each primer $(10 \mu \mathrm{M})$, $2 \mu \mathrm{L}$ of synthesized cDNA and $7 \mu \mathrm{L}$ RNase free water. The cycling conditions comprised initial 10 min incubation at $95^{\circ} \mathrm{C}$, followed by 40 cycles of $95^{\circ} \mathrm{C}$ for 15 $\mathrm{s}$ and $60{ }^{\circ} \mathrm{C}$ for $1 \mathrm{~min}$.

\section{Cytotoxicity assay:}

MTT assay was performed to determine the cell toxicity of different DNA/Protamine/Cargo complexes in each group compared to untreated cells, as negative control. About $2 \times 10^{5}$ primary isolated T cells, Jurkat cells and HEK293 cells were seeded in 24 -well plates $(1 \mathrm{ml} /$ well $)$ and incubated for $24 \mathrm{~h}$, prior to experiment. Cells were incubated with protamine, TurboFect, lipofectamine, DNA /Protamine/TurboFect and DNA/Protamine/Lipofectamine complexes for $24 \mathrm{~h}$ respectively. Then the medium was removed and replaced with $50 \mu 1(4,5-$ dimethylthiazol-2-yl)-2,5-diphenyltetrazolium bromide) tetrazolium (MTT) (Sigma-USA) diluted in RPMI 1640 (final concentration $0.5 \mathrm{mg} / \mathrm{ml}$ )). After $4 \mathrm{~h}$ 
incubation, formazan crystals were solubilized with $500 \mu$ of dimethylsulfoxide (DMSO), and the absorbance measured at $570 \mathrm{~nm}$ in a Microplate Reader (Eppendorf, Germany). The cell viability (\%) was calculated using following formula:

Cell viability $(\%)=($ Absorbance treated cells/Absorbance untreated control cells $) \times 100$

Cells which were incubated with culture media alone considered as control groups.

\section{Statical analysis:}

The data were presented as mean \pm SD and analyzed by SPSS software version 22 . The statistical significance was determined using student t-test and the $\mathrm{p}$ values $\leq 0.05$ was considered significant.

\section{Results:}

\section{Protamine significantly enhance complex surface positive charge}

After incubation a fixed quantity of DNA with increasing amounts of protamine, the complete retardation of supercoiled and relaxed forms of DNA was found at and above N/P ratios 5:1 (Fig.1).

This result showed the complete masking of DNA at this optimal N/P ratio.

On the other hand, the surface charge of different complexes was measured and the Zeta potential of DNA/TurboFect, DNA/Lipofectamine2000, DNA/Protamine/ TurboFect and DNA/Protamine/Lipofectamine 2000 were $1.2 \mathrm{mV}, 10.9 \mathrm{mV}, 22.2$ $\mathrm{mV}$ and $25.3 \mathrm{mV}$ respectively. 
Data showed that protamine significantly enhance DNA/Cargo complex surface positive charge.

Primary $T$ cells were transfected effectively by DNA/Protamine/Cargo complexes

Flow cytometry analysis revealed that TurboFect and Lipofectamine 2000 transfection efficiency was insufficient in primary $\mathrm{T}$ cells as well as Jurkat cell line.

While, the transfection rate was significantly enhanced when protamine was applied in DNA/Cargo complex formation in primary T cell, Jurkat and HEK-293 cell lines (Fig.2,3).

The mean value of GFP relative quantitation illustrated that expression level of GFP was significantly increased in DNA/Protamine/Cargo transfected T cells compared with control cells as shown in Fig. 4.

\section{Cytotoxicity assay:}

Cell viability following incubation of cells with different complexes showed no significant difference in cell viability among different cells (Fig.5).

\section{Discussion:}

Optimization of gene-delivery strategies to lymphocytes for an effective genetic manipulation in primary human $\mathrm{T}$ cell is a major challenge in fundamental $\mathrm{T}$ cell studies and clinical immunotherapy researches (15). 
Data extracted from prior studies provided noticeable evidences for the need to overcome the current barriers leading to low efficiency of gene transfer into primary human T cells $(10,16)$.

The development of an effective non-viral gene delivery method for clinical applications depends on the ability of genes to escape from lysosomal degradation and poor nuclear translocation which limit foreign DNA expression in the transfected cells $(4,16-18)$.

It is well documented that lysosomal degradation can be avoided by employing various approaches such as the proton sponge effect, that has been observed in some cationic polymers including polyethylenimine (PEI) and polyamidoamine (PAMAM) and pH-sensitive membrane lytic peptides (19).

However, the efficient nucleus targeting remains a barrier in the transfection procedure (20).

DNA condensation with a cationic nuclear targeting signal peptide, such as TAT oligomer and a tetramer of the SV40 T-antigen-derived nuclear localization signal (NLSSV40) led to increase in the nuclear membrane translocation $(21,22)$.

Protamine as a cationic peptide that has been approved by FDA is widely used in DNA condensing, nuclear targeting and the trans-gene expression enhancement.

Interestingly, the low transfection efficiency of protamine/DNA complex was documented in the previous studies due to strong hydrophilicity of protamine, which 
limits the complex to cross the cellular membrane (11). Therefore, there is absolutely essential to facilitate an electrostatic interaction between protamine and the negative charge on the cell membrane.

A standard commercial transfection reagent such as TurboFect or Lipofectamine 2000 with a safe usage profile is an alternative for current transfection strategies which primarily rely on physical methods and viral vector-based gene transfer systems. According to low transfection efficiency of conventional methods in human primary $\mathrm{T}$ cells, recent studies have focused on efforts in order to increase the level of transfection rate in these cells (23).

In this study, a combination of protamine and these transfection reagents was used to form a stable and positively charged cargo for DNA delivery into the human primary $\mathrm{T}$ cells.

Our results demonstrated that protamine at optimal N/P ratio is able to condense DNA efficiently without any cytotoxic effect on the primary human $\mathrm{T}$ cells. The results also revealed the DNA/Protamine/Cargo complexes are able to effectively transfect human primary T cells as well as Jurkat and HEK293 cell lines. The results of the present study suggest pre-condensation of DNA enhances the transfection rate of human primary T cells using TurboFect and Lipofectamine 2000 thus could be a promising non-viral gene delivery strategy to achieve a safe, rapid, cost effective 
and efficient system to further be applied in gene therapy and $\mathrm{T}$ cell manipulation methods like CAR T cell therapy.

\section{Conflict of Interest:}

The authors declare that they have no conflict of interest.

Author contribution statements: H.K, M.T and I.R. conceived of the presented idea. I.R. developed the theoretical framework; I.R wrote the article and H.K revised final version of article.

\section{Funding:}

This study was financially supported by grant No 970703 of the Biotechnology Development Council of the Islamic Republic of Iran.

\section{Summary points:}

- T lymphocytes are considered as a hard to transfect primary cells.

- T cells are resistant to common chemical transfection reagents, such as Lipofectamine 2000, polyfect and TurboFect.

- These chemical transfection reagents are less popular in the case of $\mathrm{T}$ cell transfection due to their low transfection efficiency.

- Protamine, a FDA approved cationic nuclear protein, improve DNA packaging and deliver DNA to the nucleus due to containing four nuclear localization sequences (NLS).

- DNA/Protamine/Cargo complexes effectively transfect human primary T cells. 


\section{References:}

1. Jin C, Fotaki G, Ramachandran M, Nilsson B, Essand M, Yu D. Safe engineering of CAR T cells for adoptive cell therapy of cancer using long-term episomal gene transfer. EMBO molecular medicine. 2016;8(7):702-11.

2. Hu W-S, Pathak VK. Design of retroviral vectors and helper cells for gene therapy. Pharmacological Reviews. 2000;52(4):493-512.

3. Wang GP, Garrigue A, Ciuffi A, Ronen K, Leipzig J, Berry C, et al. DNA bar coding and pyrosequencing to analyze adverse events in therapeutic gene transfer. Nucleic acids research. 2008;36(9):e49-e.

4. El-Aneed A. An overview of current delivery systems in cancer gene therapy. Journal of Controlled Release. 2004;94(1):1-14.

5. Schambach A, Baum C. Clinical application of lentiviral vectors-concepts and practice. Current gene therapy. 2008;8(6):474-82.

6. Gresch O, Engel FB, Nesic D, Tran TT, England HM, Hickman ES, et al. New non-viral method for gene transfer into primary cells. Methods. 2004;33(2):151-63.

7. Ledley FD. Non-viral gene therapy. Current Opinion in Biotechnology. 1994;5(6):626-36.

8. Stroh T, Erben U, Kühl AA, Zeitz M, Siegmund B. Combined pulse electroporation-a novel strategy for highly efficient transfection of human and mouse cells. PloS one. 2010;5(3):e9488.

9. Zhang Z, Qiu S, Zhang X, Chen W. Optimized DNA electroporation for primary human T cell engineering. BMC Biotechnol. 2018;18(1):4.

10. Zhao N, Qi J, Zeng Z, Parekh P, Chang C-C, Tung C-H, et al. Transfecting the hard-to-transfect lymphoma/leukemia cells using a simple cationic polymer nanocomplex. Journal of controlled release. 2012;159(1):104-10.

11. Motta S, Brocca P, Del Favero E, Rondelli V, Cantù L, Amici A, et al. Nanoscale structure of protamine/DNA complexes for gene delivery. Applied Physics Letters. 2013;102(5):053703.

12. Chen J, Yu Z, Chen H, Gao J, Liang W. Transfection efficiency and intracellular fate of polycation liposomes combined with protamine. Biomaterials. 2011;32(5):1412-8.

13. Tsuchiya Y, Ishii T, Okahata Y, Sato T. Characterization of protamine as a transfection accelerator for gene delivery. Journal of bioactive and compatible polymers. 2006;21(6):519-37.

14. Vighi E, Montanari M, Ruozi B, lannuccelli V, Leo E. The role of protamine amount in the transfection performance of cationic SLN designed as a gene nanocarrier. Drug delivery. 2012;19(1):1-10.

15. Liu L, Johnson C, Fujimura S, Teque F, Levy JA. Transfection optimization for primary human CD8+ cells. Journal of immunological methods. 2011;372(1-2):22-9.

16. Zhao Y, Zheng Z, Cohen CJ, Gattinoni L, Palmer DC, Restifo NP, et al. High-efficiency transfection of primary human and mouse $\mathrm{T}$ lymphocytes using RNA electroporation. Molecular therapy. 2006;13(1):151-9.

17. De llarduya CT, Arangoa M, Moreno-Aliaga M, Düzgüneş N. Enhanced gene delivery in vitro and in vivo by improved transferrin-lipoplexes. Biochimica et Biophysica Acta (BBA)-Biomembranes. 2002;1561(2):209-21.

18. Jin C, Fotaki G, Ramachandran M, Nilsson B, Essand M, Yu D. Safe engineering of CAR T cells for adoptive cell therapy of cancer using long-term episomal gene transfer. EMBO Mol Med. 2016;8(7):70211.

19. Cervia LD, Chang C-C, Wang L, Yuan F. Distinct effects of endosomal escape and inhibition of endosomal trafficking on gene delivery via electrotransfection. PloS one. 2017;12(2):e0171699.

20. Hébert E. Improvement of exogenous DNA nuclear importation by nuclear localization signalbearing vectors: a promising way for non-viral gene therapy? Biology of the Cell. 2003;95(2):59-68. 
21. Akita H, Kurihara D, Schmeer M, Schleef M, Harashima H. Effect of the compaction and the size of DNA on the nuclear transfer efficiency after microinjection in synchronized cells. Pharmaceutics. 2015;7(2):64-73.

22. Masuda $\mathrm{T}$, Akita $\mathrm{H}, \mathrm{Harashima} \mathrm{H}$. Evaluation of nuclear transfer and transcription of plasmid DNA condensed with protamine by microinjection: the use of a nuclear transfer score. FEBS letters. 2005;579(10):2143-8.

23. Basiouni S, Fuhrmann H, Schumann J. High-efficiency transfection of suspension cell lines. Biotechniques. 2012;53(2):1-4. 


\section{Figures legend:}

Fig.1. Gel retardation assay of Protamine/DNA complexes at various N/P ratios showed the complete retardation of DNA at and above N/P ratios 5:1. The values mentioned corresponding with the N/P ratios of Protamine/DNA in a $20 \mu \mathrm{L}$ reaction.

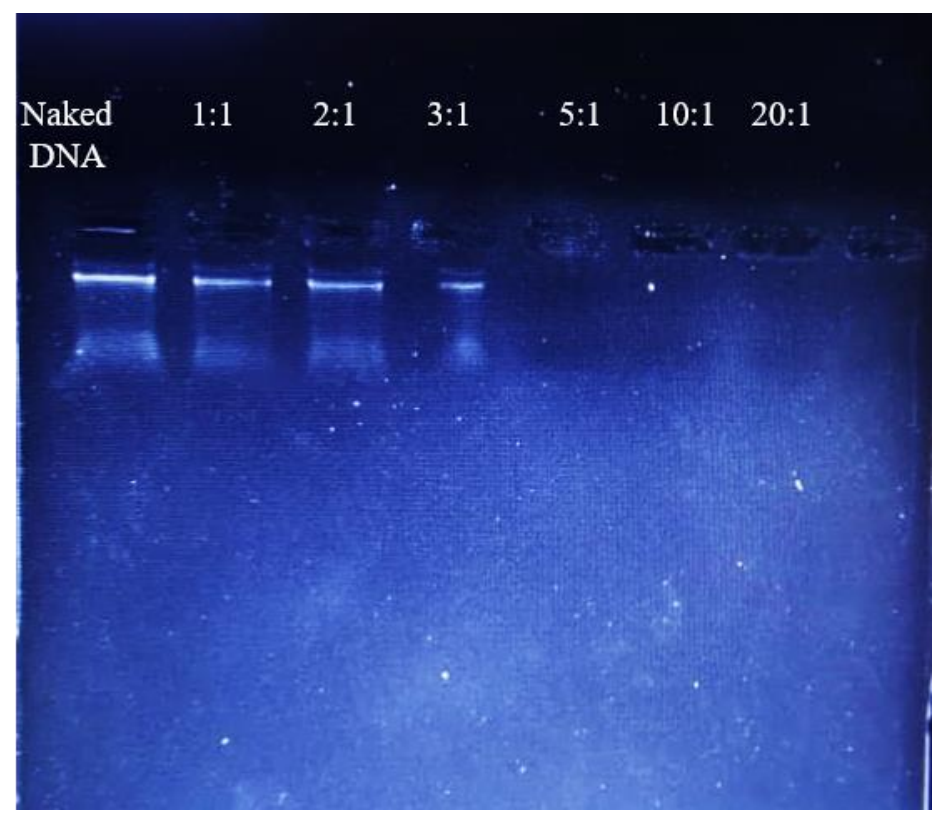


Fig.2. Protamine enhanced In vitro transfection efficiency of TurboFect and Lipofectamine 2000.

A. The fluorescent microscopy images of human primary $\mathrm{T}$ cells transfected by Lipofectamine 2000 (a), DNA/Protamine/Lipofectamine 2000 (b), TurboFect(c) and DNA/Protamine/TurboFect complex (d).

B. Flowcytometry results of human primary T cells a) untransfected b) transfected by TurboFect, c) transfected by Lipofectamine 2000, d) transfected by DNA/Protamine/TurboFect complex and e) transfected by DNA/Protamine/Lipofectamine 2000.
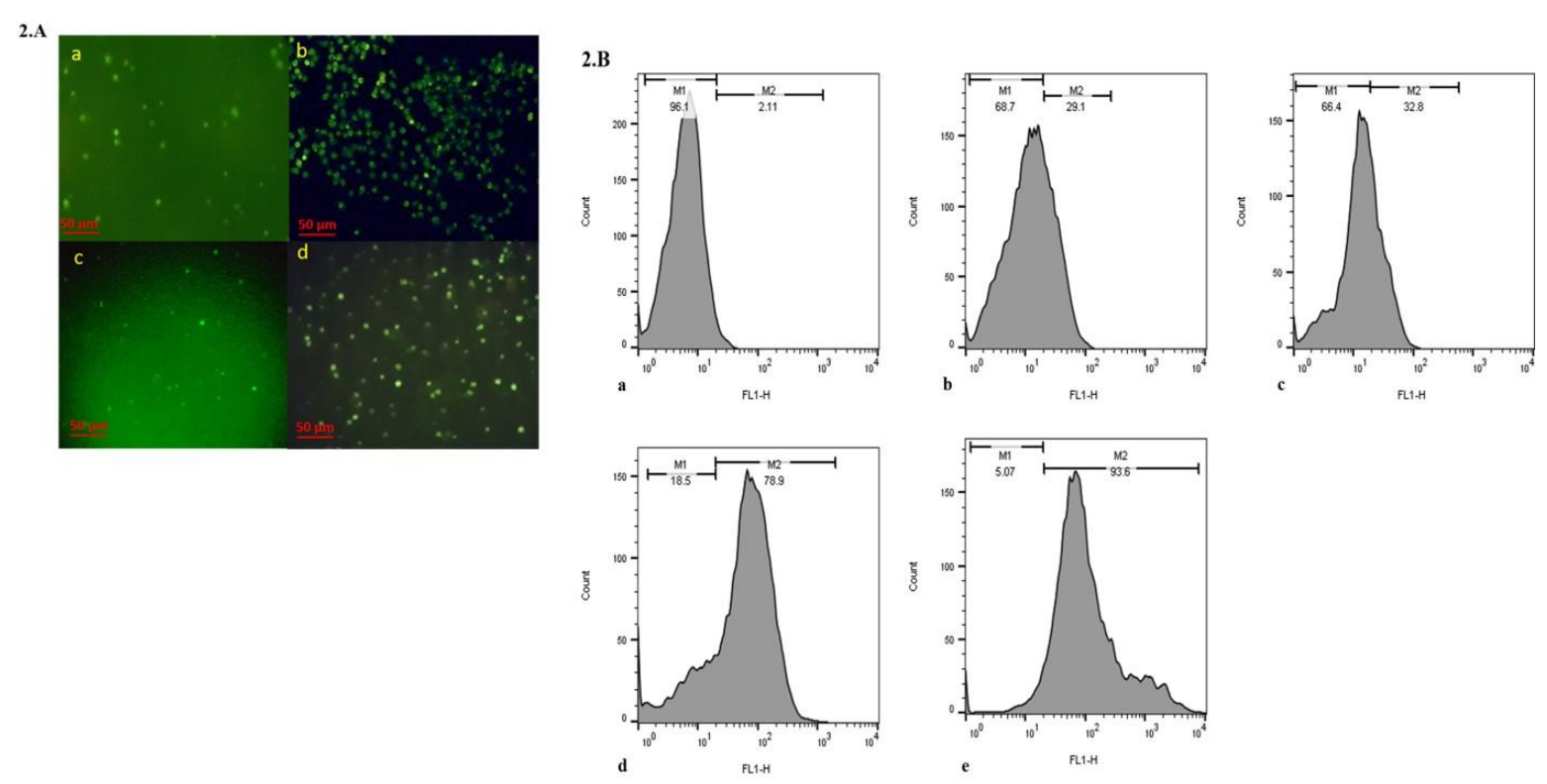
Fig.3.GFP expression was assessed in different cells using flowcytometry analysis.

There is a significant increase in the transfection rate in the case of

DNA/Protamine/Cargo complexes especially for T and Jurkat cells $(\mathrm{P}<0.001)$.

The error bars reflect the mean $\pm S D$, with $n=3$.

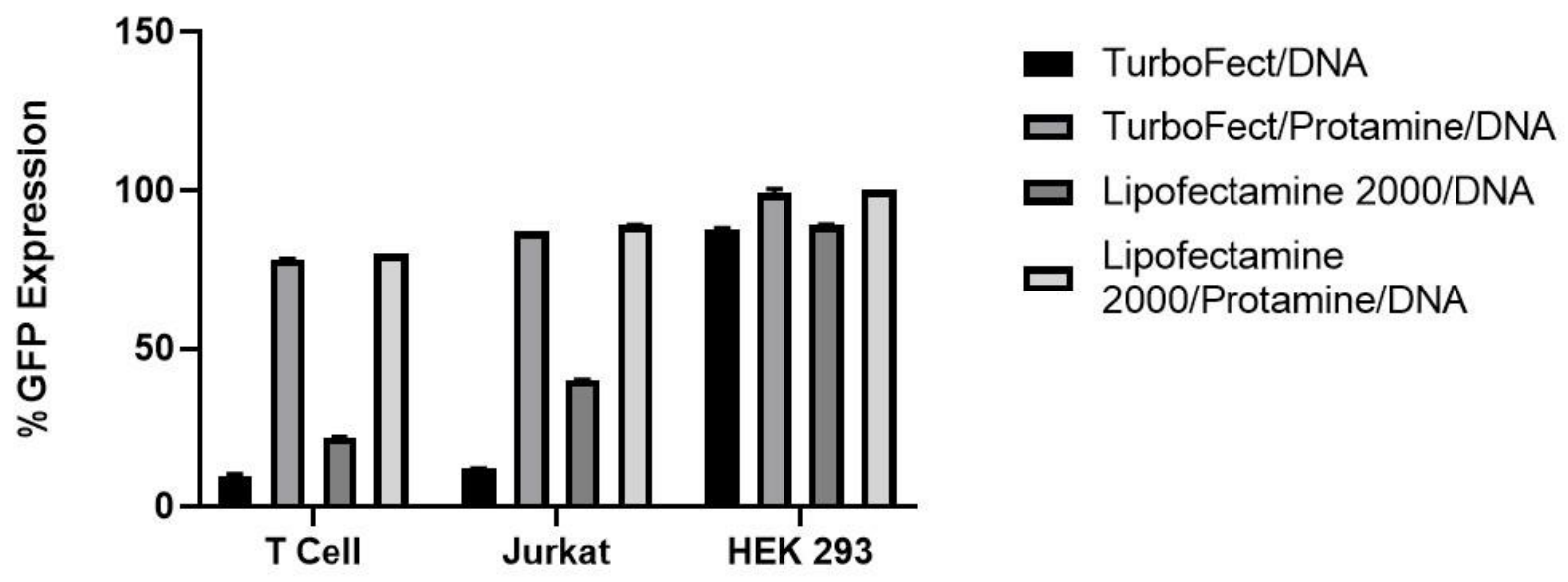


Fig.4. Real-time polymerase chain reaction (PCR) results. GFP expression level was significantly increased in transfected cells with DNA/Protamine/Cargo (*P < 0.05. $* * \mathrm{P}<0.001 . * * * \mathrm{P}<0.05$.) as illustrated in the graph.

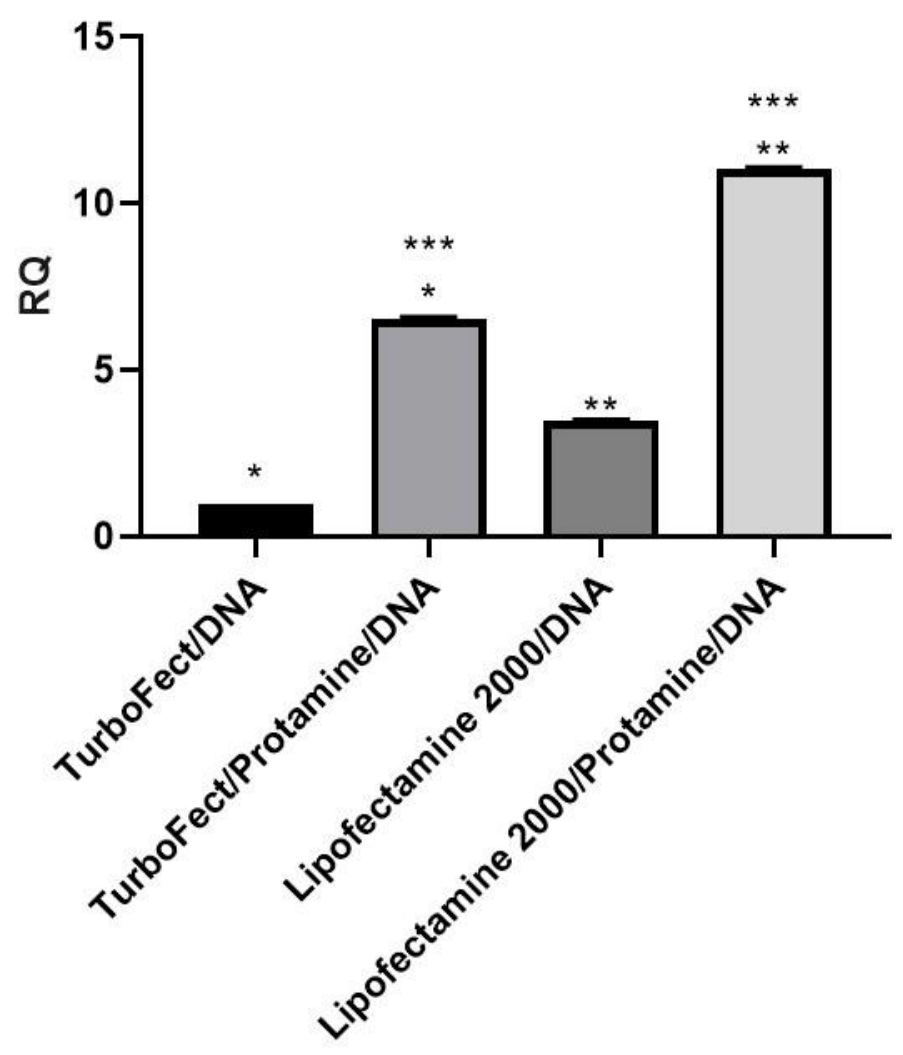


Fig.5. MTT assay results show no significant difference in cell viability between different groups with respect to the untreated control. The results shown are the mean \pm SD of three separate experiments done in duplicate $(\mathrm{P}>0.05)$.

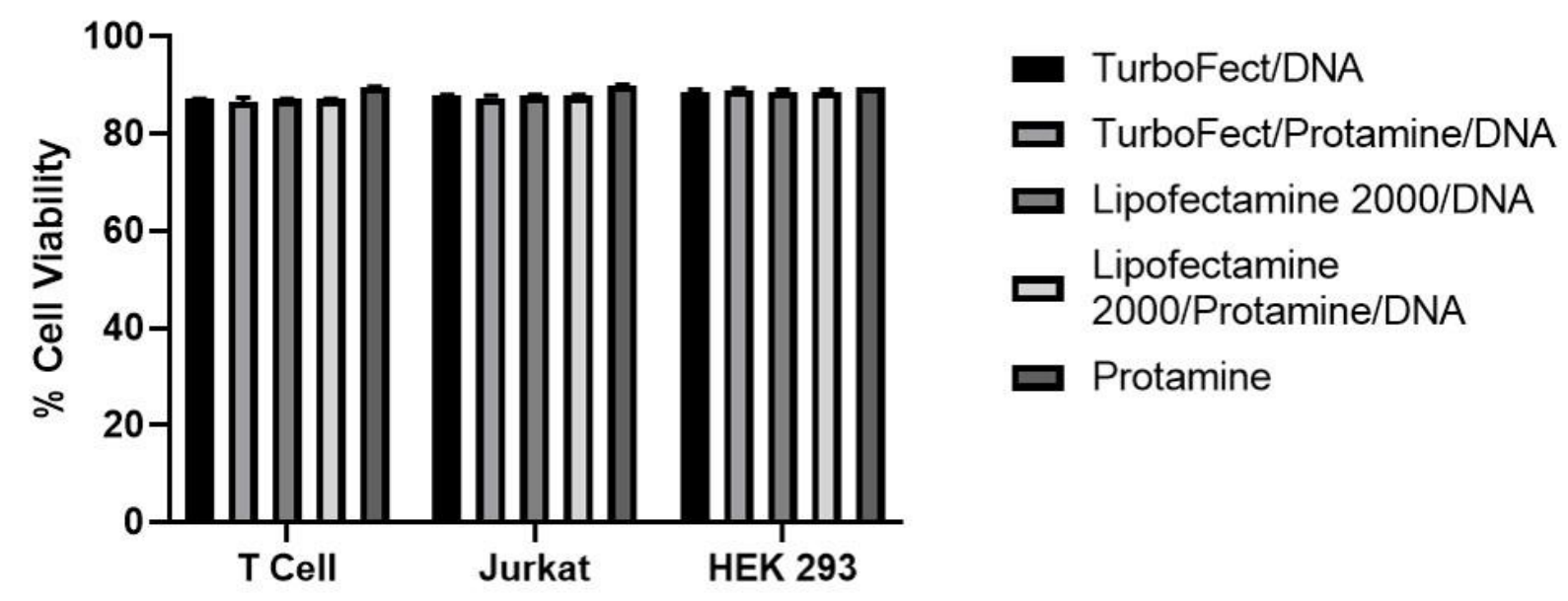

